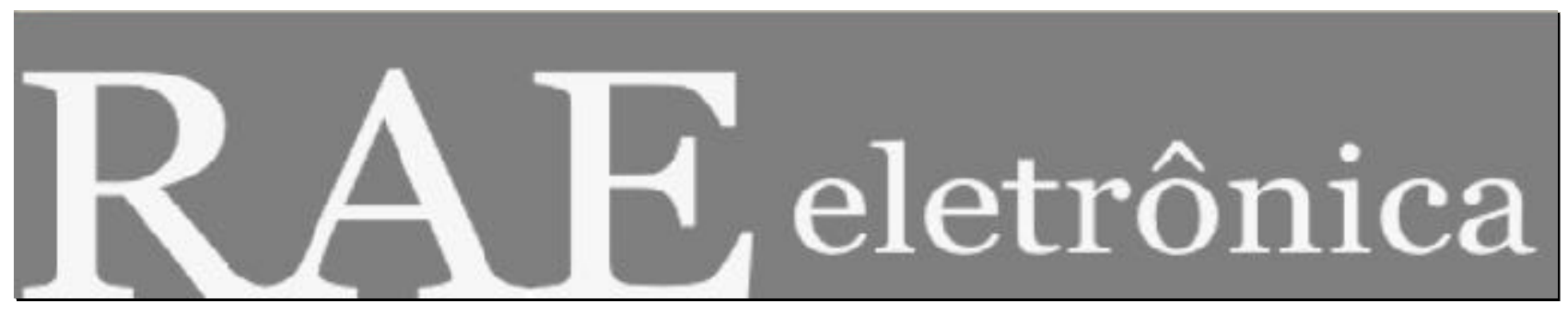

\title{
AGENTES DE SOFTWARE: DELEGANDO DECISÕES A PROGRAMAS
}

\section{Por:}

\section{Edmilson Lucena Néri}

RAE-eletrônica, v. 4, n. 1, Art. 3, jan./jul. 2005

http://www.rae.com.br/eletronica/index.cfm?FuseAction=Artigo \&ID=2095\&Secao=ARTIGOS \&Volu $\mathrm{me}=4 \&$ Numero $=1 \& A$ no $=2005$

CCopyright, 2005, RAE-eletrônica. Todos os direitos, inclusive de tradução, são reservados. É permitido citar parte de artigos sem autorização prévia desde que seja identificada a fonte. A reprodução total de artigos é proibida. Os artigos só devem ser usados para uso pessoal e nãocomercial. Em caso de dúvidas, consulte a redação: raeredacao@fgvsp.br.

A RAE-eletrônica é a revista on-line da FGV-EAESP, totalmente aberta e criada com o objetivo de agilizar a veiculação de trabalhos inéditos. Lançada em janeiro de 2002, com perfil acadêmico, é dedicada a professores, pesquisadores e estudantes. Para mais informações consulte o site www.rae.com.br/eletronica.

\section{RAE-eletrônica}

ISSN 1676-5648

(C2005 Fundação Getulio Vargas - Escola de Administração de Empresas de São Paulo.

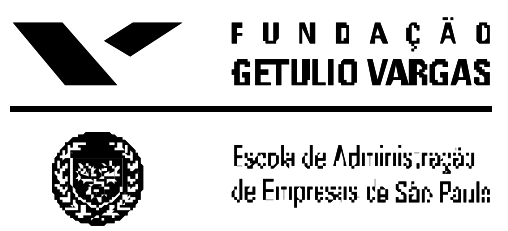




\title{
RESUMO
}

Há anos que a Tecnologia da Informação vem sendo investigada nas escolas de Administração brasileiras. Alguns temas relevantes, no entanto, ainda não receberam forte atenção. Este estudo apresenta o conceito de agentes de software e suas aplicações. Será mostrado o porquê de tanto entusiasmo na associação dos agentes, em especial, ao comércio eletrônico. Da busca por produtos e fornecedores a melhores decisões, agentes de software representam uma leitura obrigatória para quem busca entender os benefícios, desafios e cuidados que os meios de comunicação e os sistemas de informação proporcionam aos negócios. Comentários serão feitos sobre publicações encontradas em periódicos, livros e anais de congressos internacionais sobre o tema, bem como alguns trabalhos brasileiros que seguem a mesma linha. Através desta compilação crítica bibliográfica, deseja-se estimular uma maior discussão nas escolas e empresas brasileiras sobre o tema.

\section{PALAVRAS-CHAVE}

Agentes de software, tomada de decisão, comércio eletrônico.

\begin{abstract}
Information Technology has been investigated by academics at Brazilian Business Schools for a long time. Some themes related to this issue have not been discussed in Brazilian academy as much as they have in academies abroad. This text is concerned about presenting the concept of software agents and their application in Business, mainly in commerce. Throughout the text, it will be shown why software agents have made so much success within electronic trade. From product or service searches through making better decisions, software agents are a necessary reading for anyone who is trying to understand the benefits, challenges and difficulties that business is facing with communication media and information systems. Some publications from international journals, books and proceedings of academic meetings are reviewed as well as some Brazilian works. The purpose of this paper is to stimulate a greater discussion about software agents in Brazilian business schools and companies.
\end{abstract}

\section{KEYWORDS}

Software agents, decision making, electronic commerce. 


\section{INTRODUÇÃO}

Afinal, o que são agentes de software? Não há consenso sobre essa resposta ainda. Podem ser vistas várias definições na literatura, cada uma tentando adequar-se aos propósitos daqueles que a define.

Em Turban et al. (2000, p.497) há uma coletânea de conceitos. Para citar alguns:

Um agente é alguma coisa que pode ser vista como percebe ndo seu ambiente através de sensores e que age neste ambiente através de seus atuadores (RUSSEL e NORVIG, 1995).

Um agente inteligente é um software que assiste a pessoas e age em seus lugares. Agentes inteligentes permitem que pessoas deleguem trabalhos que poderiam ser feitos por agentes de software. Agentes podem, assim como assistentes podem, automatizar tarefas repetitivas, lembrar coisas que você esqueceu, sumariar inteligentemente dados complexos, aprender por você e até fazer recomendações para você (GILBERT, 1997).

Uma boa discussão sobre o conceito de agentes também pode ser encontrada em Franklin e Graesser (1996).

Um agente autônomo é um sistema situado dentro e uma parte de um ambiente que sente esse ambiente e age sobre ele, através do tempo, realizando sua própria agenda e assim afetando o que ele sentirá no futuro (FRANKLIN e GRAESSER, 1996).

Entretanto, os próprios autores reconhecem que essa definição é muito ampla. Há uma enorme quantidade de entidades que se encaixam na definição acima. Para definir subtipos de agentes, os autores enumeraram propriedades para diferenciá-los, sugerindo uma taxonomia de agentes inspirada na taxonomia dos seres vivos.

Observe-se que foi mencionado o conceito "agente autônomo" sem haver distinção ao conceito ma is simples: agente. Para muitos, o termo "autônomo" é redundante, pois definem autonomia como uma das propriedades fundamentais de uma entidade para que seja considerada um agente.

Deve-se manter em mente que um agente não necessariamente é um software. Liang e Huang (2000), por exemplo, definem agente como sendo uma pessoa ou um negócio autorizado a agir no lugar de outra. Já em desenvolvimento de software, um agente é um programa de computador que pode operar autonomamente e efetuar tarefas singulares sem a direta supervisão humana (HOFFMAN e NOVAK, 1996).

De forma geral, agentes de software podem ser considerados programas aos quais são delegadas tarefas, entendendo-se delegação como uma transferência de poder de decisão. Assim, é possível imaginar agentes de software decidindo qual a melhor compra a ser feita, quais os produtos interessantes para oferecer a um cliente, quais os momentos adequados para venda ou compra de ações, etc. 
Concomitantemente ao crescimento da importância de agentes de software, cresce a necessidade do uso de técnicas de decisão e negociação. Como todo software, agentes são formalizações do conhecimento humano, codificadas em linguagem de computadores. Sem bons mecanismos de decisão, os agentes não serão bons tomadores de decisão e, conseqüentemente, delegar tarefas para eles não parece uma boa prática.

\section{SISTEMAS MULTIAGENTES}

Outra propriedade importante de um agente é a sua comunicação com seus pares. Alguns autores incluem em suas definições tal propriedade, como em: "componentes de software que se comunicam com seus colegas através de uma expressiva linguagem de comunicação de agentes" (GENESERETH, 1994).

Sistemas multiagentes, conforme Stone e Veloso (1997), constituem uma disciplina da Inteligência Artificial que proporciona os princípios para construção de sistemas complexos, envolvendo múltiplos agentes e mecanismos para coordenação do comportamento inteligente de um conjunto dos mesmos.

A última definição tem um viés claro da Inteligência Artificial, mas sistema multiagente pode ser entendido como um sistema que envolve mais de um agente autônomo, cooperando entre si, para que o sistema consiga atingir os seus objetivos. Para cooperarem entre si, necessita haver comunicação.

Como forma de facilitar a interoperabilidade entre os agentes, recomenda-se a utilização de linguagens padrão de comunicação. Um exemplo de uma dessas linguagens é a ACL (Agent Communication Language), que é composta por três componentes: seu vocabulário, uma linguagem anterior chamada KIF (Knowledge Interchange Format) e uma outra linguagem chamada KQML (Knowledge Query and Manipulation Language). Uma mensagem ACL é uma expressão KQML em que os argumentos são termos ou sentenças em KIF formadas por palavras do vocabulário ACL (GENESERETH, 1994).

Há também o conceito de sociedade de agentes. Assim como os seres humanos, os agentes podem ser organizados em sociedades. Cada um dos agentes pode ter seu papel nessas comunidades. Respeitandose as regras ou "leis" dessa sociedade, pode-se acrescentar, retirar ou substituir agentes, sem que seja necessário "pará-la".

\section{APLICAÇÕES DE AGENTES DE SOFTWARE NO COMÉRCIO}

Muitas são as possíveis aplicações para agentes de software. Quando se têm tarefas passíveis de automação, de natureza distribuída, que exigem alta comunicação entre as partes, que normalmente têm diversas especializações; então, muitas vezes, o uso de agentes é uma boa solução computacional para o problema. 
Edmilson Lucena Néri

Agentes de software podem ser aplicados a todas as áreas onde seja útil a aplicação de um comportamento personalizado, executado de forma contínua e autônoma. Quanto mais tempo e dinheiro possam ser economizados pela automação, quanto menos riscos estejam envolvidos por se ter uma solução subótima e maior seja a importância de não perder oportunidades, mais apropriado tornase o uso de agentes (PATTIE, GUTTMAN e MOUKAS, 1999).

Muitos esforços foram e vêm sendo feitos na aplicação de agentes no comércio eletrônico. Estar ciente desses esforços é de fundamental importância para entender as transformações que vem acontecendo, bem como para prospectar o uso dessa tecnologia nos negócios. Como forma de ilustrá-los, o Modelo CBB (Consumer Buying Behavior) será usado.

Seguindo o Modelo CBB, pode-se encontrar, em quase todos seus estágios, espaço para a utilização de agentes (PATTIE, GUTTMAN e MOUKAS, 1999). A seguir, há exemplos do uso de agentes para os estágios de identificação de necessidade, busca de produto, busca por fornecedor e negociação. As etapas de compra: entrega, serviço e avaliação, não estão contempladas nesta revisão.

Hernandez e Sen (2000), chamam atenção para a incapacidade dos consumidores varejistas estarem atualizados sobre as novidades e tendências de mercado. Sugeriram um agente de software que ajuda o consumidor na escolha de uma compra, através de duas funções básicas: seleção dos produtos e sugestão de produtos alternativos. Na primeira função, o agente verifica se as restrições informadas para a escolha de um produto são factíveis. O agente retorna alguns produtos que poderiam satisfazer as necessidades do consumidor, caso o mesmo relaxasse em algumas das suas restrições. Na segunda função, de acordo com o desejo do consumidor, o agente pode informar produtos alternativos. Por exemplo, se o consumidor deseja presentear um amigo que gosta de escutar música em um CD-Player portátil, o agente pode sugerir um MP3-Player portátil, como alternativa àquele produto. A arquitetura do agente apresentado tem cinco principais módulos: interface, motor de recuperação de dados, gerador de restrições de mercado, "cruzador" de restrições e um módulo de análise.

Na mesma linha, Dutta, Debnath e Sen (2001), centram as atenções para os problemas dos consumidores ao tentar comprar algo por meio eletrônico. Em geral, uma busca por um produto leva a um retorno de muitas ofertas - ficando difícil analisar todas - de nenhuma ou poucas. A quantidade retornada dificilmente é satisfatória. A sugestão do artigo é a construção de agentes compradores que auxiliam os consumidores, através de reformulações de comandos de busca, quando:

a) não há retorno. As restrições da busca, por exemplo, podem ter sido não factíveis, como no caso de se desejar um produto de alta qualidade com um preço muito inferior aos ofertados pelo mercado. $\mathrm{O}$ agente pode sugerir um produto similar, de qualidade inferior, ao preço desejado;

b) há muitos retornos. O agente solicita ao usuário que refine sua busca, através da especificação de subcategorias.

Os agentes também têm a funcionalidade de sugerir produtos alternativos, mesmo quando o número de retornos de um comando de busca parece razoável.

Em uma edição especial do Decision Support Systems, que trata exclusivamente de agentes e modelos de e-business, Yuan (2002) apresenta uma "máquina" de comparação de compras que apresenta aos compradores um ranking com os produtos disponíveis. Há um mecanismo de aprendizado que busca aproximar a decisão da ordenação de preferências feita pelo software - inspirada na Teoria da Utilidade Multiatributo - com as preferências verdadeiras do comprador. Além da busca e ordenação dos 
produtos disponíveis procurados, a "máquina" também sugere produtos semelhantes que podem servir como substitutos.

Na etapa de negociação do modelo CBB, Liang e Huang (2000) sugerem um framework para a aplicação de agentes no suporte ao comércio eletrônico. Eles identificaram seis tipos de comércio, divididos em dois grupos: bilaterais e trilaterais. No grupo dos bilaterais existem dois envolvidos: o vendedor e o comprador. Nos trilaterais ainda há um terceiro, o corretor (broker). No primeiro gr upo, os mais comuns são: permuta (barter), barganha (bargain), licitação (bidding) e contrato (contract). No segundo tipo, os mais comuns são: leilão (auction) e compensação (clearing).

No seu framework, os autores estruturam um sistema multiagente em que cada um dos tipos tem um agente associado. Há um agente chamado "Market Maker" que escolhe, de acordo com as requisições do usuário, qual dos tipos de comércio supracitados é o adequado para a comercialização de determinado produto.

Ainda com foco no segme nto business-to-consumer, com a preocupação de munir com agentes apenas os consumidores, Preist, Byde e Bartolini (2001) trazem uma análise sobre a eficiência no uso de agentes de software em leilões do tipo inglês (a mais alta oferta ganha). Os autores observaram que vários produtos semelhantes são leiloados em diversos sites diferentes e, até mesmo, em diferentes "salas" dos mesmos sites. Acreditaram que o fato dos agentes ficarem presos a um só leilão diminui a eficiência da compra. Para provar a teoria, criourse um sistema em que o agente participa em vários leilões ao mesmo tempo. Como esperado, houve um aumento de eficiência para o comprador e, aparentemente paradoxal, também para o mercado. Contudo, segundo os resultados, à medida que aumentam os compradores munidos de tais mecanismos, só os vendedores aumentam sua eficiência.

Há uma limitação para o sistema relatado no artigo: os leilões têm que ser concorrentes, ou seja, acontecem ao mesmo tempo. Para leilões que começam em diferentes tempos, necessita-se de um mecanismo onde se possa avaliar se uma oferta atual é melhor que uma possível futura oferta em outro leilão. O caminho apontado para solucionar tal questão é um mecanismo de aprendizagem que utiliza a teoria da utilidade esperada para avaliar a possibilidade de um leilão mais recente ser mais eficiente que um leilão mais antigo ou vice-versa.

\section{SISTEMAS MULTIAGENTES PARA O COMÉRCIO}

Guttman e Maes (1998) descrevem alguns sistemas multiagentes de negociação de compras. Dividemnos em dois tipos: os que negociam competitivamente e aqueles que negociam cooperativamente. $\mathrm{Na}$ negociação cooperativa, citam duas formas de praticá-la: por funções multiatributos de utilidade e pelo método, em inglês, Distributed Contraint Satisfaction, que analisa a decisão qualitativamente.

Muitas pesquisas concentram se na construção de protocolos de negociação entre agentes para o comércio. O sistema Kasbah (CHAVEZ e MAES, 1996) implementa um protocolo simples para um sistema multiagente que tem três tipos de componentes: agentes de compra, de venda e o Marketplace. Esse último é o mediador e controlador de todo o sistema. É o Marketplace que combina compradores e vendedores que desejam comercializar os mesmos produtos. A fase de negociação, entretanto, é feita 
diretamente pelos agentes de compra e venda. Só depois de terminado o processo, com ou sem sucesso, que os agentes retornam a comunicação com o Marketplace, registrando os acontecimentos.

Uma extensão do modelo Kasbah pode ser visto em De Paula (2000), que apresenta um modelo original de negociação bilateral, introduzindo várias facilidades de uma negociação real, como a sugestão de produtos alternativos, geração de ultimatos e acordos locais em negociação de múltiplos atributos. As principais discussões deste trabalho foram: como modelar propostas, como avaliá-las, quais ações estão disponíveis para um negociador autônomo e qual ele deve tomar a cada rodada. A modelagem do sistema foi feita através da Metodologia Gaia (WOOLDRIDGE, JENNINGS E KINNY, 1999; WOOLDRIDGE, JENNINGS E KINNY, 2000). A dúvida que ficou na leitura do trabalho foi se a existência anunciada e prevista de ultimato pode levar a uma estratégia diferente de negociação que, possivelmente, venha prejudicar todo o processo.

Outros protocolos de negociação foram sugeridos, como, por exemplo, em Soo (2000), que também estabelece uma terceira parte na negociação.

Faraco (1998) sugeriu uma arquitetura de agentes para negociação, visando a utilização no comércio eletrônico. Consiste, assim como o Kasbah, de três tipos de agentes (clientes, fornecedores e roteadores) e suas inter-relações. Os atributos dos produtos em negociação são preço e quantidade. Os agentes fornecedores trabalham diretamente com as bases de dados dos fornecedores e utilizam regras preestabelecidas de negociação.

Uma outra arquitetura de um sistema multiagente para compra e venda é a relatada em Giese (1998). Tem-se um sistema que busca contemplar todo o processo de compra (em uma visão entre consumidores finais e varejistas), desde a pesquisa de mercado, para o comprador (consumidor), ao acompanhamento pós- venda, para o vendedor. Há um agente que avalia e ordena as alternativas de compra, através de tomada de decisão difusa. A decisão de comprar ou não, todavia, não é feita pelo agente. O s istema apóia a decisão, não a toma.

Para o processo de licitação, Kern (1998) propôs uma estrutura multiagente, buscando automatizá-lo. A escolha da melhor alternativa é baseada no preço. Das propostas que atendem a todos os pré-requisitos, a com o menor preço será a escolhida.

Ainda em compras e vendas, Karacapilidis e Moraïtis (2001) idealizaram um sistema multiagente onde o agente comprador "vive", mesmo após o término de uma compra. $\mathrm{Na}$ visão dos autores, criam-se empregados artificiais. Esses agentes êm também a capacidade de sugerir produtos, de forma próativa, para seus "empregadores". Os autores utilizaram alguns recursos para modelar o sistema, como mostrar as arquiteturas dos agentes ou utilizar os diagramas de Interação e de Atividades da UML (do inglês, Unified Modelling Language, a linguagem de modelagem de sistemas mais usada atualmente). Mais precisamente, usoutse uma extensão da UML para uma boa representação. Os mecanismos de decisão são um pouco diferentes entre os agentes compradores e vendedores. O algoritmo de decisão utiliza pesos para cada um dos atributos de compra, estabelecidos pelos usuários responsáveis.

Em Neri (2002), vê-se uma proposta de sistema multiagente para compras de suprimentos de informática de uma grande empresa que, pelo seu porte, pode exigir de seus fornecedores adesão a esse sistema. Algoritmos de decisão da melhor compra foram desenvolvidos e implementados, através de funções multiatributos de valor e de negociação colaborativa.

Há também, na literatura, sistema multiagente para compra e venda que foi pensado para toda a cadeia de suprimentos (CHEN et al., 1999). Cada vez que há necessidade de se comprar um suprimento, um 
Edmilson Lucena Néri

agente é delegado para fazer tal compra. Essa necessidade percorre toda a cadeia, criando, também, uma cadeia de agentes. Assim como um agente comprador escolhe o melhor agente fornecedor, um agente fornecedor também tem a capacidade de escolher com quais agentes compradores negociar.

\section{CUIDADOS PARA O COMÉRCIO ELETRÔNICO}

Quando alguma empresa resolve vender produtos pela Internet, ela terá de enfrentar novo desafios. Um grande problema é a concorrência, que, na Internet, torna-se ainda mais perigosa, devido à facilidade do consumidor "ir" de um lugar para outro - está tudo à distância de um "cliq ue".

Um poderoso aliado para pesquisar os melhores produtos e os melhores preços para os consumidores é o agente de software, como vem sendo discutido neste texto. Não só pela capacidade de busca e sugestão de produtos, mas, em especial, pela capacidade de decisão.

Em Conway e Koehler (2000), há uma excelente discussão sobre as possibilidades advindas do uso de tal tecnologia. Inicialmente, comentam o uso de agentes que buscam, em vários sites, um determinado produto, classificando, por preço, a lista dos sites. Ao perceberem os problemas causados por tais tipos de mecanismos, os desenvolvedores dos sites resolveram dificultar seu uso. A estratégia utilizada foi tornar gráficas todas as informações sobre busca no site e sobre os produtos. Assim, esperava-se que só os usuários humanos pudessem entender, uma vez que os primeiros usuários "agentes de software" faziam inferências textuais pelo próprio código-fonte das páginas (HTML, por exemplo). Para a infelicidade dos desenvolvedores dos sites, surgiram agentes que interpretam gráficos, através de mecanismos de Inteligência Artificial, bem como simulavam o uso do teclado e do mouse, de forma que não se perceba que não é um ser humano que está "visitando" tal site.

Como se não fosse suficiente o problema com a concorrência, os autores despertam para um problema ainda maior, associado ao uso de agentes de software no comércio: melhores decisões. Muitas vezes, os seres humanos são incapazes de garantir um bom mecanismo de decisão. Com a implementação de mecanismos automatizados de decisão em agentes, os consumidores podem atingir maior qualidade em suas escolhas, como já discutido em alguns casos para leilões.

$\mathrm{O}$ artigo traz um exemplo do uso de agentes, munidos de mecanismos de decisão, que mostram o potencial desta associação. Foi desenvolvido um agente de software para jogar "blackjack" em cassinos on-line. O "blackjack" é um jogo de cartas que tem um valor esperado positivo, quando se joga de forma ótima. Isto significa dizer que se um jogador faz jogadas ótimas (as melhores possíveis com as informações disponíveis), ao passar do tempo, ele ganha mais do que perde. Jogadores muito experientes apenas se aproximam de jogar otimamente. Assim, os cassinos ganham, em média, mais do que perdem com os apostadores, mesmo com a existência de alguns jogadores experientes. Entretanto, para um software, não é difícil jogar de forma ótima o "blackjack". Para comprovar tal afirmação, construiu-se um agente de software que tem algoritmos para jogadas ótimas no "blackjack". Como resultado, os autores do artigo provaram que seu agente levaria o cassino à bancarrota.

O que se pode aprender em toda essa discussão é que fazer comércio por meios eletrônicos implica cuidados novos, pois não só as distâncias desaparecem, como se abrem as portas para mecanismos mais precisos de decisão e negociação. 


\section{CONSIDERAÇÕES FINAIS}

Foram descritos, neste trabalho, os principais conceitos e experiências recentes relacionados a agentes de software. O fato de delegar tarefas a programas de computador tem implicações realmente novas e surpreendentes. Para o comércio, por exemplo, não se trata apenas de um novo meio para se fazer o que sempre se fez: comprar e vender. Fundamentalmente, a forma em que as pessoas pesquisam, decidem e negociam produtos pode ser profundamente modificada. Empresas podem aumentar seu leque de potenciais parceiros em uma negociação, uma vez que estas tarefas podem ser automatizadas.

Novas formas das empresas interagirem com seus consumidores estão sendo pensadas. No Media Lab do MIT (Massachusetts Institute of Technology, nos EUA), por exemplo, pesquisa-se soluções de hardware e software para tornar possível aos clientes a localização de serviços e produtos nas proximidades em que se encontram fisicamente. Mais uma vez, soluções ssando agentes de software aparecem (TEWARI, YOULL e MAES, 2002). O que se pode observar é que, no mundo tão repleto de informações e possibilidades, mecanismos que auxiliem, ou mesmo tomem decisões para as pessoas, serão bem comuns de serem encontrados.

Com o tema "agentes" em evidência, a importância da criação de bons mecanismos de decisão torna-se imperativo. Cabe, principalmente, aos estudiosos e praticantes da Administração, pública e privada, formalizar seus conhecimentos, bem como sugerir novas abordagens para possíveis implementações dos agentes. A compreensão das possibilidades dessa tecnologia, bem como suas ameaças, são também imperativas na formação daqueles que desejam estar preparados para fornecer, comprar, sugerir e negociar produtos e serviços para empresas parceiras e consumidores finais que, como visto, já têm - e terão ainda mais - ferramentas para melhorar suas decisões.

Durante todo este texto, pesquisas e experiências no uso de agentes de software foram relatadas, de forma a estimular pesquisadores e profissionais da Administração a buscarem mais informações sobre o tema, adequando-os as suas respectivas áreas de interesse. Agentes de software já são realidade e, cada vez mais, estarão presentes no apoio e na própria tomada de decisão. Aqueles que dominarem melhor a formalização dos processos decisórios, associada ao uso dessa tecnologia, estarão mais bem munidos para explorar as possibilidades que a Tecnologia da Informação traz aos negócios. As referências bibliográficas apresentadas são um bom começo. Boa leitura!

\section{REFERÊNCIAS BIBLIOGRÁFICAS}

CHAVEZ, A.; MAES, P. Kasbah: An agent marketplace for buying and selling goods. In: First International Conference on the Practical Application of Intelligent Agents and Multi-Agent Technology, Londres: 1996. 
CHEN, Y. et al. A negotiation-based multiagent system for supply chain. In: AGENTS 99 Workshop on Agent Based Decision-Supported for Managing the Internet-Enabled Supply-Chain, Seattle: p. 1520, 1999.

CONWAY, D. G.; KOEHLER, G. J. Interface agents: caveat mercator in electronic commerce. Decision Support Systems, v. 27, p. 355-366, 2000.

DE PAULA, G. E. Modelo de negociação bilateral para comércio eletrônico. 2000. Dissertação (Mestrado em Ciência da Computação) - Universidade Federal de Pernambuco, Recife.

DUTTA, P. S., DEBNATH, S., SEN, S. A Shopper's assistant. In: The Fifth International Conference on Autonomous Agents (ACM AGENTS'01), Montreal: p. 59-60, 2001.

FARACO, R. A. Uma arquitetura de agentes para negociação dentro do domínio do comércio eletrônico. 1998. Dissertação (Mestrado em Ciência da Computação) - Universidade Federal de Santa Catarina, Florianópolis.

FRANKLIN, S.; GRAESSER. A. Is it an agent, or just a program? A taxonomy for autonomous agents. In: The Third International Workshop on Agents Theories, Architectures, and Languages, Verlag: 1996.

GENESERETH, M. R. Software agents. Communications of the ACM, v. 37, n. 7, 1994.

GIESE, L. F. Estrutura de agentes para os processos de compra e venda utilizando tomada de decisão difusa. 1998. Dissertação (Mestrado em Ciência da Computação) - Universidade Federal de Santa Catarina, Florianópolis.

GILBERT, D. Intelligent agents: the right information at the right time. IBM White Paper. [S.1.], 1997.

GUTTMAN, R. H.; MAES, P. Cooperative vs. competitive multi-agent negotiations in retail electronic commerce. In: Second International Workshop on Cooperative Information Agents, Paris: 1998.

HERNANDEZ, K. , SEN, S. A buyer's agent. In: Proceedings of the Fourth International Conference on Autonomous Agents (ACM AGENT'00), Barcelona: p. 152-162, 2000.

HOFFMAN, D. L., NOVAK, T.P. Marketing in hyper-media computer-mediated environments: conceptual foundations. Journal of Marketing, v. 60, n. 2, p. 50-68, 1996.

KARACAPILIDIS, N., MORAÏTIS, P. Intelligent agents for an artificial market system In: The Fifth International Conference on Autonomous Agents (ACM AGENTS'01), Montreal: p. 592-599, 2001.

KERN, E. Uma estrutura de agentes para o processo de licitação. 1998. Dissertação (Mestrado em Ciência da Computação) - Universidade Federal de Santa Catarina, Florianópolis.

LIANG, T. P.; HUANG, J. S. A framework for applying intelligent agents to support electronic trading. Decision Support Systems, v. 28, p. 305-317, 2000.

NERI, E. L. Agentes de software nas empresas: um estudo de caso na área de compras. 2002. Dissertação (Mestrado em Administração), Universidade Federal do Rio Grande do Sul, Porto Alegre. 
PATTIE, M., GUTTMAN, R. H., MOUKAS, A. G. Agents that buy and sell: transforming commerce as we know It. Communications of the ACM, v. 43, 1999.

PREIST, C., BYDE, A., BARTOLINI, C. Economic dynamics of agents in multiple auctions. In: The Fifth International Conference on Autonomous Agents (ACM AGENTS'01, 2001), Montreal: p. 545$551,2001$.

RUSSEL, J., NORVIG, P. Artificial intelligence: a modern approach. New Jersey: Prentice Hall, 1995.

SOO, V. W. Agent negotiation in trusted third part mediated uncertain games. In: The Fourth International Conference on Autonomous Agents (ACM AGENT'00), Barcelona: p. 265-266, 2000.

STONE, P., VELOSO, M. Multiagent systems: a survey from a machine learning perspective. $C M U C S$ technical report number CMU-CS-97-193, 1997.

TEWARI, G.; YOULL, J.; MAES, P. Personalized location-based brokering using an agent-based intermediary architecture. Decision Support Systems, v. 34, p. 127-137, 2002.

TURBAN, E.; LEE, J. K.; KING, D., CHUNG, M. Electronic commerce: a managerial perspective, Primeira Edição, New Jersey: Prentice Hall, 2000.

WOOLDRIDGE, M., JENNINGS, N. R., KINNY, D. A methodology for agent-oriented analysis and design. In: The Third International Conference on Autonomous Agents (ACM AGENTS'99), Seattle: $\mathrm{p}$. 69-76, 1999.

WOOLDRIDGE, M., JENNINGS, N. R., KINNY, D. The gaia methodology for agent-oriented analysis and Design. Journal of Autonomous Agents and Multi-Agent Systems, v. 3, n. 3, p. 285-312, 2000 .

YUAN, S. A personalized and integrative comparasion-shopping engine and its applications. Decision Support Systems, v. 34, p. 139-156, 2002.

\section{Artigo recebido em 20.08.2003. Aprovado em 22.07.2004.}

\section{Edmilson Lucena Néri}

Mestre em Administração pela EA/UFRGS

Interesses de pesquisa nas áreas de sistemas de informação, agentes de software, teorias da decisão e negociação.

E-mail: elneri@ig.com.br

Endereço: Av. Nego, 585, apto 302, Tambaú, João Pessoa - PB, 58039-920. 